\title{
Perron and Frobenius Meet Carathéodory
}

\author{
Márton Naszódi \\ Dept. of Geometry, Loránd Eötvös University \\ MTA-ELTE Lendület Combinatorial Geometry Research Group \\ Budapest, Hungary \\ marton.naszodi@math.elte.hu \\ Alexandr Polyanskii \\ Laboratory of Combinatorial and Geometric Structures \\ Moscow Institute of Physics and Technology (National Research University) \\ Dolgoprudny, Russia \\ Laboratory 4, Institute for Information Transmission Problems RAS \\ Moscow, Russia \\ Caucasus Mathematical Center, Adyghe State University, Maykop, Russia \\ alexander.polyanskii@yandex.ru
}

Submitted: Nov 4, 2020; Accepted: Jun 24, 2021; Published: Jul 16, 2021

(C) The authors. Released under the CC BY-ND license (International 4.0).

\begin{abstract}
We present a new approach of proving certain Carathéodory-type theorems using the Perron-Frobenius Theorem, a classical result in matrix theory describing the largest eigenvalue of a matrix with positive entries.

At the end, we list some results and conjectures that we hope can be approached with this method.
\end{abstract}

Mathematics Subject Classifications: Primary 52A35; Secondary 15B48

\section{Introduction}

Carathéodory's Theorem [Car07] is one of cornerstones of combinatorial geometry as Eckhoff [Eck93] called it. The theorem claims that a point in the convex hull of a set $P \subseteq$ $\mathbb{R}^{d}$ is in the convex hull of at most $d+1$ points of $P$. The survey [Eck93] contains a number of generalizations of the theorem, which is closely related to a number of other classical results in convex geometry, such as Helly's and Radon's Theorems [Hel23, Rad21]. In this note, we provide a new proof of the theorem that shows a connections with a principal theorem in matrix theory, the Perron-Frobenius Theorem, and prove other known results in convexity using the same approach.

We mention a few other recent results in convex and discrete geometry where the Perron-Frobenius theory was applied. The authors of [CEKMS19] found a proof of 
the Alexandrov-Fenchel inequality using the Perron-Frobinius theorem (see the proof of Proposition 3 in [CEKMS19]). In [Pol19], the second named author of the current paper used the Perron-Frobenius theorem to bound the size of an almost equidistant diameter set of points in $\mathbb{R}^{d}$, that is, a finite set of points of diameter 1 such that among any three points there are two at distance 1. At last, we remark that the role of the Perron-Frobenius theory is crucial in the study of equiangular lines; see [JTY+19] and papers cited therein.

The paper is organized as follows. In Section 2, we introduce our tools. In Section 3, we illustrate the key idea applying it to prove Rankin's Theorem [Ran55, Theorem 1(iii, iv)]. In Section 4, we use the idea to prove Carathéodory's and Steinitz's Theorems [Car07, Ste13]. Finally, in Section 5, we list a few known facts as well as open questions as candidates to be approached with the method.

\section{Preliminaries}

\section{$2.1 \quad$ Notation}

We write $[n]=\{1, \ldots, n\}$, for a positive integer $n$. The convex hull of a finite set of points $\left\{x_{1}, \ldots, x_{n}\right\} \subset \mathbb{R}^{d}$, written $\operatorname{conv}\left\{x_{1}, \ldots, x_{n}\right\}$, is the set

$$
\left\{\sum_{i=1}^{n} \lambda_{i} x_{i}: \lambda_{i} \geqslant 0 \text { for all } i \in[n], \sum_{i=1}^{n} \lambda_{i}=1\right\} .
$$

A point $x$ of a set $P \subseteq \mathbb{R}^{d}$ is an interior point of $P$, if the set $P$ contains some open ball with center in $x$.

The spectral radius of a square matrix $A$, written $\rho(A)$, is the largest absolute value of its eigenvalues.

\subsection{Tools}

Since we do not need the most general form of the Perron-Frobenius Theorem [Per07, Fro12], we state only two of its corollaries.

Lemma 1 (Perron's Theorem). For a square matrix with positive entries, the spectral radius is an eigenvalue of multiplicity 1 , such that its eigenvector has positive entries.

Lemma 2 (Frobenius's Theorem). For a square matrix with non-negative entries, the spectral radius is an eigenvalue such that one of its eigenvectors has non-negative entries.

Also, we need two finite-dimensional versions of the Hahn-Banach Theorem in Section 4 .

Theorem 3. If the origin o of $\mathbb{R}^{d}$ does not lie in the convex hull of points $x_{1}, \ldots, x_{n} \in \mathbb{R}^{d}$, then there exists a vector $y \in \mathbb{R}^{d}$ such that $\left\langle y, x_{i}\right\rangle>0$ for all $i \in[n]$.

Theorem 4. If the origin o of $\mathbb{R}^{d}$ is not an interior point of the convex hull of points $x_{1}, \ldots, x_{n} \in \mathbb{R}^{d}$, then there exists a vector $y \in \mathbb{R}^{d}$ such that $\left\langle y, x_{i}\right\rangle \geqslant 0$ for all $i \in[n]$. 


\section{Proof of Rankin's Theorem}

We state the two parts of Rankin's Theorem as separate theorems.

Theorem 5 (Rankin). If $\left\{v_{1}, \ldots, v_{n}\right\}$ is a set of non-zero vectors in $\mathbb{R}^{d}$ such that the angle between any two of them is larger than $\frac{\pi}{2}$, then $n \leqslant d+1$.

Proof. Suppose to the contrary that $n \geqslant d+2$. Let $G$ be the Gram matrix of the vectors $v_{1}, \ldots, v_{n}$, that is, $G=\left(\left\langle v_{i}, v_{j}\right\rangle\right)_{i, j=1}^{n}$. Choose a positive $\lambda$ such that $\lambda>\left\langle v_{i}, v_{i}\right\rangle$ for all $i \in[n]$, and set $H=\lambda I_{n}-G$. The hypothesis of the theorem implies that all entries of $H$ are positive. By Lemma 1, the spectral radius $\rho(H)$ is the largest eigenvalue of $H$ of multiplicity one.

Obviously, $\operatorname{rank} G \leqslant d$ because $G$ is the Gram matrix of $d$-dimensional vectors. Hence 0 is an eigenvalue of $G$ of multiplicity at least two, that is, $\lambda$ is an eigenvalue of $H$ of multiplicity at least two. Since the Gram matrix $G$ is positive semidefinite, and thus, all its eigenvalues are non-negative, $\lambda$ must be the largest eigenvalue of $H$. Therefore, $\lambda=\rho(H)$, contradicting the fact that the multiplicity of the largest eigenvalue is one.

Theorem 6 (Rankin). If $\left\{v_{1}, \ldots, v_{n}\right\}$ is a set of non-zero vectors in $\mathbb{R}^{d}$ such that the angle between any two of them is at least $\frac{\pi}{2}$, then $n \leqslant 2 d$.

Proof. Suppose to the contrary that $n \geqslant 2 d+1$. Without loss of generality we can assume that the vectors $v_{1}, \ldots, v_{n}$ are of unit length. Let $G$ be the Gram matrix of the vectors $v_{1}, \ldots, v_{n}$. Set $H=I_{n}-G$. The hypothesis of the theorem implies that all entries of $H$ are non-negative. By Lemma 2, the spectral radius $\rho(H)$ is the largest eigenvalue of $H$.

Obviously, $\operatorname{rank} G \leqslant d$ because $G$ is the Gram matrix of $d$-dimensional vectors. Hence, 0 is an eigenvalue of $G$ of multiplicity at least $n-d$, and thus, 1 is an eigenvalue of $H$ of multiplicity at least $n-d$. Since the Gram matrix $G$ is positive semidefinite, that is, 0 is its smallest eigenvalue, 1 is the largest eigenvalue of $H$. It means that $\rho(H)=1$. Let $\lambda_{1}, \ldots, \lambda_{d}, 1, \ldots, 1$ be the eigenvalues of $H$, indexed in non-decreasing order. Therefore, we have

$$
0=\operatorname{tr}(H)=\lambda_{1}+\cdots+\lambda_{d}+(n-d), \text { and thus, }\left|\lambda_{1}+\cdots+\lambda_{d}\right|=n-d \geqslant d+1 .
$$

However, the last inequality contradicts $\left|\lambda_{i}\right| \leqslant 1=\rho(H)$ for all $i \in[d]$.

\section{Proofs of Caratheodory's and Steinitz's Theorems}

Theorem 7 (Carathéodory's Theorem). If the origin $o \in \mathbb{R}^{d}$ lies in the convex hull of points $v_{1}, \ldots, v_{n} \in \mathbb{R}^{d}$, then there is a set $J \subseteq[n]$ of size at most $d+1$ such that o lies in $\operatorname{conv}\left\{v_{j}: j \in J\right\}$.

Proof. We use induction on $n$. The base case, $n=d+1$, being trivial, it is sufficient to show that if $n>d+1$ and $o \notin \operatorname{conv}\left\{v_{i}: i \in[n] \backslash\{j\}\right\}$ for all $j \in[n]$, then $o \notin$ $\operatorname{conv}\left\{v_{i}: i \in[n]\right\}$.

By Theorem 3, for all $j \in[n]$, there is a vector $y_{j}$ such that $\left\langle v_{i}, y_{j}\right\rangle>0$ for all $i \in[n] \backslash\{j\}$. Choose a positive $\lambda$ such that $\lambda+\left\langle v_{i}, y_{i}\right\rangle>0$ for all $i \in[n]$ and set $H=\lambda I_{n}+V^{t} Y$, where $V=\left[v_{1}, \ldots, v_{n}\right]$ and $Y=\left[y_{1}, \ldots, y_{n}\right]$. By Lemma 1 , the spectral radius $\rho(H)$ is the largest eigenvalue of $H$ of multiplicity one. 
Obviously, rank $V^{t} Y \leqslant d$ because $V$ and $Y$ are $d$-by-n matrices. Hence, 0 is an eigenvalue of $V^{t} Y$ of multiplicity at least two, that is, $\lambda$ is an eigenvalue of $H$ of multiplicity at least two. It follows that $\rho(H) \neq \lambda$, so $\rho(H)>\lambda$, because the spectral radius cannot be less than a positive eigenvalue. Consider the eigenvector $x$ of the eigenvalue $\rho(H)$. By Lemma 1, its entries are positive. Therefore, we obtain

$$
\left(\lambda I_{n}+V^{t} Y\right) x=\rho(H) x, \text { and thus, } V^{t} Y x=(\rho(H)-\lambda) x .
$$

Hence, $Y x$ is a $d$-dimensional vector such that $\left\langle v_{i}, Y x\right\rangle>0$ for all $i \in[n]$, because all entries of the vector $(\rho(H)-\lambda) x$ are positive. So the points $v_{1}, \ldots, v_{n}$ lie in an open half-space bounded by a hyperplane passing through the origin. Thus, their convex hull does not contain the origin.

Theorem 8 (Steinitz's Theorem). If the origin o of $\mathbb{R}^{d}$ is an interior point of the convex hull of points $v_{1}, \ldots, v_{n} \in \mathbb{R}^{d}$, then there is a set $J \subseteq[n]$ of size at most $2 d$ such that the point o is interior of $\operatorname{conv}\left\{v_{j}: j \in J\right\}$.

Proof. Again, we use induction on $n$. The base case, $n=2 d$, being trivial, it is sufficient to show that if $n>2 d$ and, for all $j \in[n]$, the origin $o$ is not an interior point of conv $\left\{v_{i}: i \in[n] \backslash\{j\}\right\}$, then $o$ is not an interior point of conv $\left\{v_{i}: i \in[n]\right\}$.

By Theorem 4, for all $j \in[n]$, there is a non-zero vector $y_{j}$ such that $\left\langle v_{i}, y_{j}\right\rangle \geqslant 0$ for all $i \in[n] \backslash\{j\}$. If $\left\langle v_{i}, y_{i}\right\rangle \geqslant 0$ for some $i \in[n]$, then the origin is not an interior point of $\operatorname{conv}\left\{v_{1}, \ldots, v_{n}\right\}$. So, without loss of generality, we may assume that $\left\langle v_{i}, y_{i}\right\rangle=-1$ for all $i \in[n]$. Set $H=I_{n}+V^{t} Y$, where $V=\left[v_{1}, \ldots, v_{n}\right]$ and $Y=\left[y_{1}, \ldots, y_{n}\right]$. By Lemma 2, the spectral radius $\rho(H)$ is an eigenvalue of $H$.

Obviously, rank $V^{t} Y \leqslant d$ because $V$ and $Y$ are $d$-by- $n$ matrices, and hence, 0 is an eigenvalue of $V^{t} Y$ of multiplicity at least $(n-d)$, that is, 1 is an eigenvalue of $H$ of multiplicity at least $(n-d)$. Suppose that $\rho(H)=1$. Let $\lambda_{1}, \ldots, \lambda_{d}, 1, \ldots, 1$ be the eigenvalues of $H$, indexed in non-decreasing order. Hence we have

$$
0=\operatorname{tr}(H)=\lambda_{1}+\cdots+\lambda_{d}+(n-d), \text { and thus, }\left|\lambda_{1}+\cdots+\lambda_{d}\right|=n-d \geqslant d+1 .
$$

But the last inequality contradicts $\left|\lambda_{i}\right| \leqslant 1=\rho(H)$ for all $i \in[d]$. Hence $\rho(H)>1$ because the spectral radius cannot be less than a positive eigenvalue. Consider the eigenvector $x$ of the eigenvalue $\rho(H)$. By Lemma 2, its entries are non-negative entries. Therefore, we get

$$
\left(I_{n}+V^{t} Y\right) x=\rho(H) x, \text { and thus, } V^{t} Y x=(\rho(H)-1) x .
$$

Hence, $\left\langle v_{i}, Y x\right\rangle \geqslant 0$ for all $i \in[n]$. Moreover, $Y x$ is a non-zero vector, because among non-negative entries of $(\rho(H)-1) x$ there is at least one positive. So the points $v_{1}, \ldots, v_{n}$ lie in a closed half-space bounded by a hyperplane passing through the origin, that is, the point $o$ is not interior of their convex hull.

\section{Concluding Remarks}

Our proofs use induction on the number of points, but not on the dimension. As a natural question, we ask if induction can be completely avoided.

In [BBM91], A. Bezdek, K. Bezdek and E. Makai obtained the following result concerning a point in the interior of a convex polytope with few vertices. 
Theorem 9 (A. Bezdek, K. Bezdek, E. Makai). If the origin o of $\mathbb{R}^{d}$ is an interior point of the convex hull of $n$ points $v_{1}, \ldots, v_{n} \in \mathbb{R}^{d}$ with $d<n \leqslant 2 d$, then there is a set $J \subset[n]$ of size at least $\left\lceil\frac{n}{n-d}\right\rceil$ such that o lies in the relative interior of $\operatorname{conv}\left\{v_{j}: j \in J\right\}$.

A colorful generalization of Carathéodory's theorem due to Bárány [Bár82] reads as follows.

Theorem 10 (Bárány). Let $F_{1}, \ldots, F_{d+1}$ be finite sets of points in $\mathbb{R}^{d}$ such that the origin $o$ is contained in the convex hull of each. Then there is a $(d+1)$-point set $E$ in $F_{1} \cup \cdots \cup F_{d+1}$ with $E \cap F_{i}$ a singleton for each $i \in[d+1]$ such that $o \in \operatorname{conv}(E)$.

It would be interesting to see short proofs of Theorems 9 and 10 using our method based on the Perron-Frobenius Theorem.

We mention the following difficult problem. In [BKP82] (see also [BKP84]), Bárány, Katchalski and Pach showed the following Quantitative Steinitz Theorem.

Theorem 11 (Bárány, Katchalski and Pach). For every dimension d, there is a constant $r=r(d)>0$ such that if the origin centered unit ball is contained in the convex hull of points $v_{1}, \ldots, v_{n} \in \mathbb{R}^{d}$, then there is a set $J \subseteq[n]$ of size at most $2 d$ such that the origin centered ball of radius $r(d)$ is contained in $\operatorname{conv}\left\{v_{j}: j \in J\right\}$.

In [BKP82], the bound $r(d) \geqslant d^{-2 d}$ is established (in fact, a bound of order roughly $d^{-d / 2}$ is shown implicitly), and $r(d) \geqslant c d^{-1 / 2}$ is conjectured with some universal constant $c>0$. We do not know if this problem can be approached using our method.

\section{Acknowledgments}

Part of the research was carried out while the authors were members of János Pach's chair of DCG at EPFL, Lausanne, which was supported by Swiss National Science Foundation Grants 200020-162884 and 200021-175977.

MN was supported also by the National Research, Development and Innovation Fund (NRDI) grant K119670, by the ÚNKP-20-5 New National Excellence Program of the Ministry for Innovation and Technology from the source of the NRDI, as well as the János Bolyai Scholarship of the Hungarian Academy of Sciences.

AP was supported by the program "Leading Scientific Schools" through Grant No. NSh-2540.2020.1 (Theorems 5 and 6) and by the Russian Government through Megagrant No. 075-15-2019-1926 (Theorems 7 and 8).

The authors are grateful to János Pach and Imre Bárány for fruitful discussions, Galyna Livshyts for bringing [CEKMS19] to our attention and Endre Makai for sharing [BBM91] with us.

\section{References}

[Bár82] Imre Bárány, A generalization of Carathéodory's theorem, Discrete Math. 40 (1982), no. 2-3, 141-152.

[BBM91] András Bezdek, Károly Bezdek, and Endre Makai, Jr., Interior points of the convex hull of few points in $\mathbf{E}^{d}$, Monatsh. Math. 111 (1991), no. 3, 181-186. 
[BKP82] Imre Bárány, Meir Katchalski, and János Pach, Quantitative Helly-type theorems, Proc. Amer. Math. Soc. 86 (1982), no. 1, 109-114.

[BKP84] Imre Bárány, Meir Katchalski, and János Pach, Helly's theorem with volumes, Amer. Math. Monthly 91 (1984), no. 6, 362-365.

[Car07] Constantin Carathéodory, Über den Variabilitätsbereich der Koeffizienten von Potenzreihen, die gegebene Werte nicht annehmen, Mathematische Annalen 64 (1907), no. 1, 95-115.

[CEKMS19] Dario Cordero-Erausquin, Bo'az Klartag, Quentin Merigot, and Filippo Santambrogio, One more proof of the alexandrov-fenchel inequality, Comptes Rendus Mathematique 357 (2019), no. 8, 676-680.

[Eck93] Jürgen Eckhoff, Helly, Radon, and Carathéodory type theorems, Handbook of Convex Geometry, Part A, Elsevier, 1993, pp. 389-448.

[Fro12] Ferdinand Georg Frobenius, Über Matrizen aus nicht negativen Elementen, Sitzungsber. Königl. Preuss. Akad. Wiss. (1912), 456-477.

[Hel23] Eduard Helly, Über Mengen konvexer Körper mit gemeinschaftlichen Punkte, Jahresbericht der Deutschen Mathematiker-Vereinigung 32 (1923), 175-176.

$[$ JTY+19] Zilin Jiang, Jonathan Tidor, Yuan Yao, Shengtong Zhang, and Yufei Zhao, Equiangular lines with a fixed angle, 2019, arXiv:1907.12466.

[Per07] Oskar Perron, Zur Theorie der Matrices, Mathematische Annalen 64 (1907), no. 2, 248-263.

[Pol19] Alexandr Polyanskii, On almost-equidistant sets - II, The Electronic Journal of Combinatorics 26(2) (2019), \#P2.14.

[Rad21] Johann Radon, Mengen konvexer Körper, die einen gemeinsamen Punkt enthalten, Mathematische Annalen 83 (1921), no. 1-2, 113-115.

[Ran55] Robert Alexander Rankin, The closest packing of spherical caps in $n$ dimensions, Glasgow Mathematical Journal 2 (1955), no. 3, 139-144.

[Ste13] Ernst Steinitz, Bedingt konvergente Reihen und konvexe Systeme, Journal für die reine und angewandte Mathematik 143 (1913), 128-176. 